(Sari et al.)

\title{
Optimal Control on Model of SARS Disease Spread with Vaccination and Treatment
}

\author{
Ririt Andria Sari ${ }^{1 *}$, Ummu Habibah², Agus Widodo \\ ${ }^{1}$ Master Program of Mathematics, Faculty of Mathematics and Natural Sciences, University of Brawijaya, Malang, \\ Indonesia \\ ${ }^{2}$ Department of Mathematics, Faculty of Mathematics and Natural Sciences, University of Brawijaya, Malang, Indonesia
}

\begin{abstract}
The spread of SARS (Severe Acute Respiratory Syndrome) disease in a human population is one of the phenomena that can be mathematically modeled. The exposed period of SARS disease underlies the formation of the SVEIR epidemic model which is a modification of the SVIR epidemic model by adding subpopulation E (exposed). In the SVEIR model, there are two control variables in the form of vaccination and treatment which aimed to minimize exposed subpopulation, infected subpopulation, and control implementation cost. The Pontryagin's minimum principle is used to obtain optimal control and system, thus minimizing objective functional as the objective to be achieved. Furthermore, the forward-backward sweep method is used for numerical simulation in order to determine the most appropriate control strategy in a finite time. The simulation results show that implementation of both vaccination and treatment is the most effective decision making to control the spread of SARS disease.
\end{abstract}

Keywords: optimal control, Pontryagin's minimum principle, SARS.

\section{INTRODUCTION}

Infectious diseases are caused by pathogens or biological agents such as virus, bacteria, fungi, and other microorganisms. Infectious diseases can be transmitted from one individual to another through a variety of intermediate such as body fluids, feces, and tools contamined by individual infected SARS virus [1]. Susceptible individual can be infected with the SARS virus as a result of making contact with infected individual so that it can lead to new infection that will spread the disease to other susceptible individual. This event is a sign of the spread of disease in a society or country that can increase mortality in a short time. It is reported by the WHO that infectious disease contribute about $1 / 6$ of total deaths worldwide and second factor causes of death [2].

SARS is an infectious disease caused by virus. The clinical symptoms of SARS disease are fever, dry cough, shortness of breath, and other symptoms similar to pneumonia [3]. The spread of SARS disease is very rapid, progressive, and fatal. Most people with SARS disease are adults between 25-70 years, but in some cases SARS also affects children under 15 [4]. The spread of SARS disease was first detected in November 2002 in

\footnotetext{
* Correspondence address:

Ririt Andria Sari

Email : riritandria17@gmail.com

Address : Dept. Mathematics, University of Brawijaya, Veteran Malang, Malang 65145.
}

Guangdong province, China. Furthermore, SARS disease spread rapidly throughout the continent, especially in the Asia-Pacific region. In March 2003, WHO reported SARS disease caused by a virus called coronavirus or SARS-CoV [3]. That caused 774 people died and 8098 people infected SARS-coV virus [5]. The extermination of the SARS outbreak is estimated to have cost about \$ 10-30 billion [6]. However, the amount of funds spent does not necessarily overcome the disease outbreaks optimally.

The impact of infectious diseases are very harmful in a country or population, it is important to understand the dynamics of disease progression and develop the control of disease spread and consider the costs associated with control implementation. In this case, a mathematical model is needed to illustrate future disease spread by involving information in the present. Mathematical modeling is an important tool in understanding the dynamics of disease spread and decision-making processes related to the control program of a disease spread. The branch of mathematics developed to find the optimal way of controlling dynamic systems is called optimal control theory. The application of optimal control theory aims to estimate the effectiveness of various policies, control measures, and their associated costs [7].

Vaccination is a very popular control policy. Implementation of vaccination program is estimated to prevent approximately 2-3 million 
deaths each year and 25 diseases with vaccination approved by WHO. In recent decades, vaccination and some control policies such as treatment, quarantine, isolation, screening, etc. are also applied to control the spread of infectious diseases [2].

In 2017, Kumar and Srivastava added control variables to the SVIR (Susceptible Vaccinated Infected Recovered) epidemic model in order to control the spread of the disease. In addition to vaccinations, treatment is also needed to prevent the spread of disease. Therefore, vaccination and treatment control strategies are used. On the economic side, the implementation of a control strategy within a country requires substantial cost. Limited funding by health or government institutions is a matter to be considered, so Kumar and Srivastava also consider the costs in their research [2].

Some infectious diseases such as tuberculosis, influenza, measles, etc. have exposed period in the natural world. In the exposed period the susceptible individu does not show symptom of the disease and does not transmit the disease because the immune system is in good condition. This individu will stay in the exposed class during the exposed period. After the exposed period end or the immune decrease, the individu is in the class of infection and shows symptoms and then transmit the disease [8].

Epidemic models with latent stage and vaccination were investigated, expressed in the form of SVEIR namely Susceptible Vaccinated Exposed Infected Recovered [9]. Before the study, the SVEIR model has been studied to describe the spread of SARS disease [10]. However, there is no effort to control the spread of disease from those researchs. Previous research aimed to assess the potential impact of an anti-SARS vaccine is not perfect through mathematical modeling [10]. Further result show that an imperfect anti-SARS vaccine successfully reduces the spread of SARS in the community on the condition that the vaccine must have efficacy or effectiveness of at least $75 \%$ [10].

This study modifies the SVIR epidemic model into SVEIR by adding exposed subpopulation (E). This is due to the exposed period of SARS for 3-5 days [1] and the subpopulation model E is based on the study [9]. In addition to adding subpopulation $E$, it is assumed that individual infected with SARS cannot recover naturally by model [1]. This modification has a goal to make the model more real. Furthermore, two control functions that is vaccination control and treatment control are added to the SVEIR model. Vaccination is used to control the spread of SARS disease, where susceptible individual is given anti-SARS vaccine to build up immunity against SARS virus [10], and apply control policies in the form of treatment given to individuals infected with SARS. The existence of control function in this study aims to minimize exposed subpopulation and infected subpopulation so that the spread of SARS disease is not widespread and minimize the costs associated with the implementation of control. Optimal control issues resolved with the Pontryagin's minimum principle. At the end, a numerical simulation is done using the forward-backward sweep method. Furthermore, the simulation results are analyzed to determine the most effective control strategies in controlling the spread of SARS disease.

\section{MATERIALS AND METHODS}

In this study, SARS disease spread model assumed that the individual who became the object of research is human, interaction between individu occur in one population without any individu entering or leaving the population, the birth rate and the death rate are constant. The spread of SARS disease in this study focused on one population divided into 5 subpopulations, namely susceptible subpopulation $S$, vaccinated subpopulation $V$, exposed subpopulation $E$, infected subpopulation $I$, and recovered subpopulation $R$. Furthermore, several research methods are used to achieve the objectives.

\section{Literature Study}

Literature study related to the research process, such as the literature discussing the spread of disease, optimal control theory, the Pontryagin principle, and forward-backward sweep method. We also used other supporting references in problem solving in this study.

\section{Model Formulation}

Constructed spread model of infectious diseases SARS with type SVEIR. The SVEIR model is obtained from the SVIR model which added subpopulation $\mathrm{E}$ (exposed). The subpopulation model $E$ is based on the research of Li et al [9], where the susceptible individual infected with the disease due to contact with the infected individual will enter into the exposed (E) subpopulation during the exposed period. After the exposed period end, the individual begins to show clinical symptoms and has the ability to transmit the disease, so that the individual is included in the 
infected subpopulation (I). Furthermore, an assumption is added to the SVEIR model that individual infected with SARS cannot recover naturally, it's based on the model of Huang's research [1]. The addition of these assumption causes the natural recovery rate of infected individual is zero.

\section{Optimal Control Simulation}

Formulated optimal control problem and resolved with the Pontryagin's minimum principle. Based on these principle required a necessary condition that must be met. The necessary condition can be generated with the Hamiltonian $\mathrm{H}$ function. The $\mathrm{H}$ function must be optimal in order to obtain the optimal system. If the optimal system then achieved the optimal goal. The system can achieve optimal if the $\mathrm{H}$ function satisfies state equations, costate equations, and stationer condition.

Numerical simulation is performed for optimal control problem. The simulation includes simulating the spread of SARS disease when not given vaccination and treatment controls, when only vaccination control is given, when only treatment control is given, and when combination control of vaccination and treatment are given. The numerical simulation is solved using the forward-backward sweep method with Matlab software. The initial parameter values used in the simulations will be mentioned in the results and discussion. The initial parameter values are used to analyze and emphasize the simulated results obtained.

Analyzing the results of numerical simulation to find out the most effective or appropriate control strategies, thus minimize exposed subpopulation, infected subpopulation, and costs associated with the implementation of control (vaccination and treatment).

\section{RESULT AND DISCUSSION \\ SARS Epidemic Model}

In this section, the SVEIR epidemic model is formulated to illustrate the spread of SARS in a population divided into 5 subpopulations. Individu in each subpopulation interact with each other. This interaction causes the movement of individu from one subpopulation to another. The flow of individu movement from one subpopulation to another subpopulation is illustrated in Figure 1 . The arrows entered in a subpopulation indicate the presence of incoming individual, so the number of individual in the subpopulation increases. The outbound arrows of a subpopula- tion indicate the presence of an outgoing individual, so the number of individuals in the subpopulation is reduced.

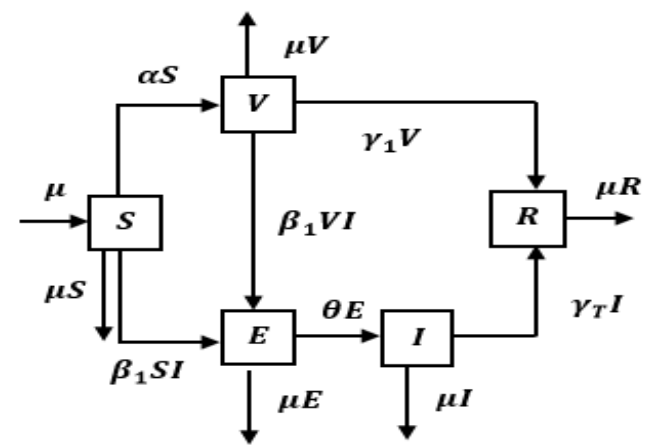

Figure 1. Compartment diagram of the SVEIR epidemic model

The number of individuals in each subpopulation may vary from time to time due to the influence of natural factors such as birth and death as well as the presence of susceptible individual into infected individual and so on, so that the number of individuals each time can be expressed $S(t), V(t), E(t), I(t), R(t)$.

The rate of susceptible subpopulation change between time $t_{0}$ and $T$ is

$$
\frac{d S}{d t}=\lim _{\Delta t \rightarrow 0} \frac{\Delta S(t)}{\Delta t} \text {. }
$$

The rate of subpopulation change also applies to the rate of change of vaccinated, exposed, infected, and recovered subpopulation. Based on Figure 1, the SARS disease spread model is expressed in the system of differential equations as follows:

$$
\begin{aligned}
& \frac{d S}{d t}=\mu-\beta S I-\alpha S-\mu S, \\
& \frac{d V}{d t}=\alpha S-\beta_{1} V I-\gamma_{1} V-\mu V, \\
& \frac{d E}{d t}=\beta S I+\beta_{1} V I-\theta E-\mu E, \\
& \frac{d I}{d t}=\theta E-\gamma_{T} I-\mu I-\sigma I, \\
& \frac{d R}{d t}=\gamma_{1} V+\gamma_{T} I-\mu R,
\end{aligned}
$$

with initial density

$$
S(0) \geq 0, V(0) \geq 0, E(0) \geq 0, I(0) \geq 0, R(0) \geq 0 \text {. }
$$

In the SVEIR model expressed in the system of equations (1), all values of the parameters are assumed to be non-negative constants. Parameter $\mu$ represents birth and mortality 
natural rate, $\beta$ is the transmission rate of disease when infected individual make contact with susceptible individual. $\beta_{1}$ represent the transmission rate of disease from individual infected with SARS against vaccinated individual who have not been immune to the disease, $\alpha$ is constant vaccination rate in susceptible subpopulation, $\gamma_{1}$ represent immunity rate of vaccinated individual so that individual become recovered individual during or after the vaccination process, $\gamma_{T}$ is treatment rate of infected individual, dan $\theta$ represent rate of exposed individual become infected individual.

Vaccination and treatment are selected as control policies because vaccination are easy obtained and applied in practice and supported by WHO [2]. Therefore, in this study, the SARS disease spread model expressed in model system (1) is modified into a model system (2) with vaccination and treatment as a control strategy to be discussed in the next chapter.

\section{Optimal Control Problem}

The SARS disease spread model with vaccination and treatment control strategies is obtained based on the system of equation (1) and the following statements.

i. Susceptible subpopulation are given antiSARS vaccine with the aim of providing immunity to susceptible individual from SARS disease, thereby preventing transmission of the disease to susceptible individual. In some diseases, constant vaccination may not be a good choice in the economic context for any country. In addition, vaccine administration for large population need substantial cost and difficult to give vaccine for all susceptible individual in the population. Therefore, it is important to know the exact level and timing of the vaccine so that maximize the vaccinated individual with minimum vaccination cost over a finite time period. According to Kumar and Srivastava [2], it can be done by changing the constant vaccination rate $\alpha$ on model (1) become $u_{1}(t)$ function as vaccination control. Due to limited fund and time for health agencies in implementing vaccination policies, then the policies should be limited in accordance with established goal. Thus, it is assumed that vaccination control is limited, i.e. $0 \leq u_{1}(t) \leq 1$ [2].

ii. Infected subpopulation are given treatment to reduce the burden of disease and control the spread of infection. Treatment programs include diagnosis, drug administration, hospitalization, and other medical services. Similar to vaccination programs, treatment programs for infected individuals also require a large cost and must be minimized. Therefore, treatment rate $\gamma_{T}$ on model (1) become $u_{2}(t)$ as treatment control and assumed that $0 \leq u_{2}(t) \leq 1$ [2].

Thus, SARS disease spread model with vaccination and treatment control is as follows:

$$
\begin{aligned}
& \frac{d S}{d t}=\mu-\beta S I-u_{1}(t) S-\mu S, \\
& \frac{d V}{d t}=u_{1}(t) S-\beta_{1} V I-\gamma_{1} V-\mu V, \\
& \frac{d E}{d t}=\beta S I+\beta_{1} V I-\theta E-\mu E, \\
& \frac{d I}{d t}=\theta E-u_{2}(t) I-\mu I-\sigma I, \\
& \frac{d R}{d t}=\gamma_{1} V+u_{2}(t) I-\mu R,
\end{aligned}
$$

with a set of control function $U$, i.e.

$$
U=\left\{u_{1}(t), u_{2}(t) \mid 0 \leq u_{i}(t) \leq 1, i=1,2, t \in[0, T]\right\},
$$

$t$ represent time and $T$ represent final time for control strategy SARS disease. The final time for each disease is varied and the implementation of the control strategy depend on the objective of the control policy. Implementation of control will be stopped when the objective have been achieved.

Application of optimal control theory in case of SARS disease spread with vaccination and treatment aims to minimize exposed subpopulation, infected subpopulation, and costs associated with control implementation. This objective is expressed in an objective function $J$, i.e.

$$
\begin{array}{r}
J\left[u_{1}(t), u_{2}(t)\right]=\int_{0}^{T}\left[E+I+w_{1} u_{1}^{2}(t)\right. \\
\left.+w_{2} u_{2}^{2}(t)\right] d t,
\end{array}
$$

with positive constant $w_{1}$ represent the weight of vaccination and $w_{2}$ represent the weight of treatment should be minimized. The objectives expressed in equation (3) have the constraints expressed in the model systems (2). These constraints illustrate the pattern or model of the spread of SARS disease in a population where the 
initial value of each subpopulation is known i.e. $S(0) \geq 0, V(0) \geq 0, E(0) \geq 0, I(0) \geq 0, R(0) \geq 0$.

The initial value is used to determine future value after vaccination and treatment control are given, so it can be seen the effectiveness of the methods to prevent the spread of SARS disease. If the functional value in equation (3) has a minimum functional value, then the control strategy is more effective.

The functional value of $J$ can be minimized by applying the Pontryagin's minimum principle. On these principle, a necessary condition must be met. These necessary condition may be generated by the Hamiltonian function (denoted $\mathrm{H}$ ) defined as follows:

$$
\begin{aligned}
H(t, \vec{x}, \vec{u}, \vec{\lambda})= & f(t, \vec{x}, \vec{u}) \\
& +\sum_{i=1}^{n} \lambda_{i}(t) g_{i}(t, \vec{x}, \vec{u}),
\end{aligned}
$$

Where $f$ is the integrand of the objective function $\mathrm{J}$ in equation (3), $g_{i}$ is the right-hand side of the equations in system 2 , and $\lambda_{i}$ is the adjoint functions dependent on the number of state variables. The state variable in this research are 5 $(S, V, E, I, R)$. Furthermore, $H$ function must be optimal to obtain the optimal $J$ value, that is state equation, costate equation, and stationary condition must be satisfied.

The state equation is satisfied when the partial derivative of $H$ function to adjoint function $\lambda$ yields equations in system (2). The costate equation is satisfied when the partial derivative of the $H$ function for each state variable is negative. The stationary condition is obtained when the partial derivative of the $H$ function to the control variable $u_{1}(t)$ and $u_{2}(t)$ is zero, so that the optimal control of $u_{1}^{*}(t)$ dan $u_{2}^{*}(t)$, that is

$$
\begin{aligned}
& u_{1}^{*}(t)=\min \left\{\max \left\{0, \frac{\left(\lambda_{1}-\lambda_{2}\right) S}{2 w_{1}}\right\}, 1\right\}, \text { and } \\
& u_{2}^{*}(t)=\min \left\{\max \left\{0, \frac{\left(\lambda_{4}-\lambda_{5}\right) I}{2 w_{2}}\right\}, 1\right\},
\end{aligned}
$$

with $\lambda_{1}, \lambda_{2}, \lambda_{4}$, and $\lambda_{5}$ are adjoint functions. If optimal control $u_{1}^{*}(t)$ and $u_{2}^{*}(t)$ are substituted in the state and costate equations then the optimal system is obtained which can optimize the objective function $J$.

\section{Numerical Simulation}

In this section, numerical simulation results are used to analyze the impact of control policy strategies on disease dynamic and related costs of control implementation. Numerical simulations of systems (2) and (3) use forwardbackward sweep method in the MATLAB software. Initial values and parameter values are used for numerical simulations are presented in table 1.

Table 1. Initial Values and Parameter Values

\begin{tabular}{ccccc}
\hline$S(0)$ & $V(0)$ & $E(0)$ & $I(0)$ & $R(0)$ \\
\hline 0.8 & 0.04 & 0.08 & 0.04 & 0.04 \\
\hline$\mu$ & $\gamma_{1}$ & $\beta$ & $\beta_{1}$ & $\theta$ \\
\hline 0.00005 & 0.04 & 0.3 & 0.01 & 0.02 \\
\hline
\end{tabular}

In order to analyze the utility of control policy, three numerical control strategies are performed, i.e.

strategy A: implementation of a single control policy that only applies vaccination to prevent the spread of SARS disease,

strategy B: implementation of a combination control policy: vaccination and treatment to prevent the spread of SARS disease, and

strategy C: implementation of a single control policy that only applies treatment to prevent the spread of SARS disease.

The discussion of this paper includes numerical simulation by implementing control strategies $A, B, C$, and without vaccination or treatment control. The numerical simulation result in Figure 2 show the changes in behavior of exposed subpopulation (E) when applied control strategy $A$, $\mathrm{B}, \mathrm{C}$ and the weight of costs $w_{1}=w_{2}=20$. The number of exposed subpopulation has increased each time when no vaccination or treatment control are provided. After control strategy $A$ is applied, the number of exposed subpopulation decreases faster than without control. The number of exposed subpopulation also decreased when applied control strategy B, i.e. the combination control strategy of vaccination and treatment are applied.

When strategy $C$ is applied, the number of subpopulation $\mathrm{E}$ shows no difference when it is given only treatment control and no control. This means, the implementation of the treatment control strategy only (strategy $\mathrm{C}$ ) is ineffective to 
reduce the number of exposed subpopulation. Thus, the implementation of vaccination control only can minimize the number of exposed subpopulation. However, it is better to be given a combination of controls i.e. vaccination and treatment to suppress the number of exposed subpopulation.

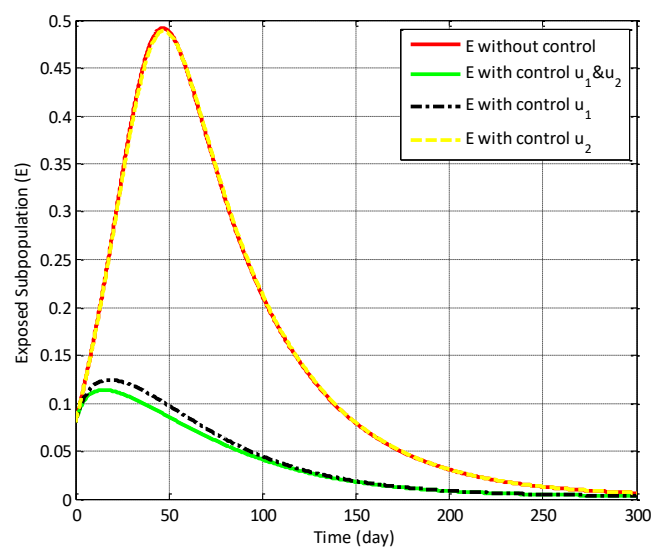

Figure 2. Density of Exposed Subpopulation (E) with Control Strategy A, B, C, and Without Control

The change in the number of infected subpopulations (I) at any time in Figure 3 is same as the number of exposed subpopulation. The number of subpopulation I decreased significantly when the combination control of vaccination and treatment are given. The strategy is more effective than either vaccination control strategy only or treatment control strategy only.

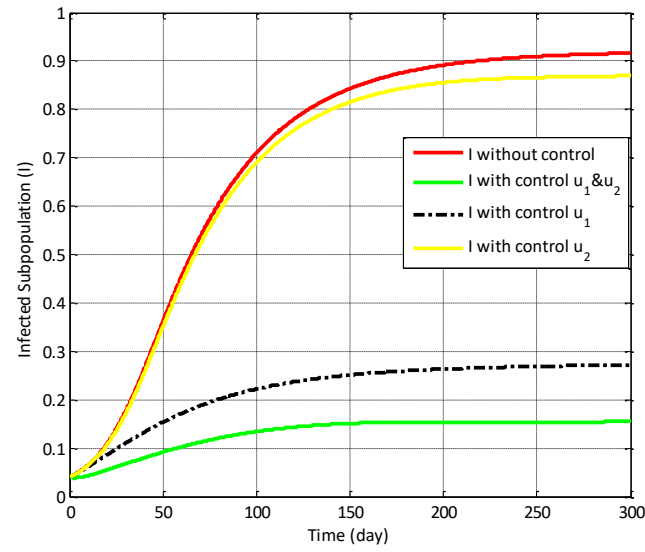

Figure 3. Density of Infected Subpopulation (I) with Control Strategy A, B, C, and Without Control

From the Figures 2 and 3, it can seen that the number of exposed (E) and infected (I) subpopulations reach the least value when combination control of vaccination and treatment is given. In other words, control strategy $B$ is more effective than control strategy $A$ and $C$. Level of vaccina- tion and treatment are given every time (day), it can be seen in Figure 4. At first, vaccination control is given equal to 0.16 and treatment control is 0.07 . When the number of exposed and infected subpopulations decrease, vaccination and treatment control are lowered to zero. That means, control of vaccination and treatment are stopped.

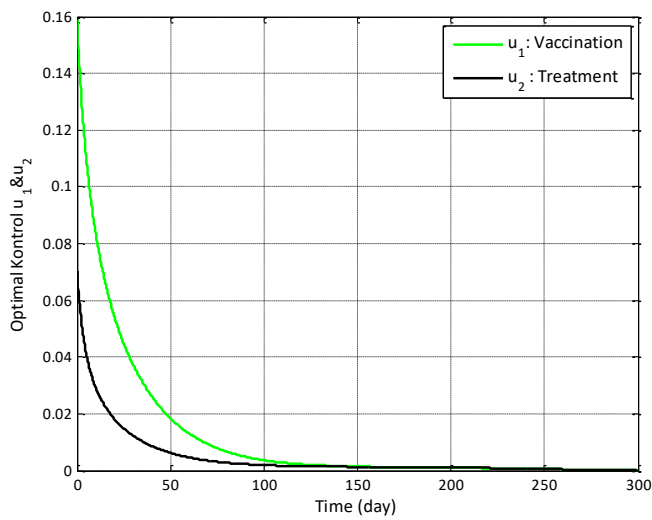

Figure 4. Vaccination control $u_{1}$ and treatment control $u_{2}$ are given every time (day)

Figure 5 shows the effect of the weight of vaccination cost $w_{1}$ and the weight of treatment cost $w_{2}$ on the number of exposed subpopulation (E), $w_{1}=w_{2}=2000$. When the weight of vaccination and treatment costs is enlarged a hundred times from the initial weight, the number of exposed subpopulation is not much different from the number of exposed subpopulation when no control is given. This is due to very small vaccination and treatment controls that is equal to $8.391 \times 10^{-3}$ and $2.96 \times 10^{-3}$, as shown in Figure 7.

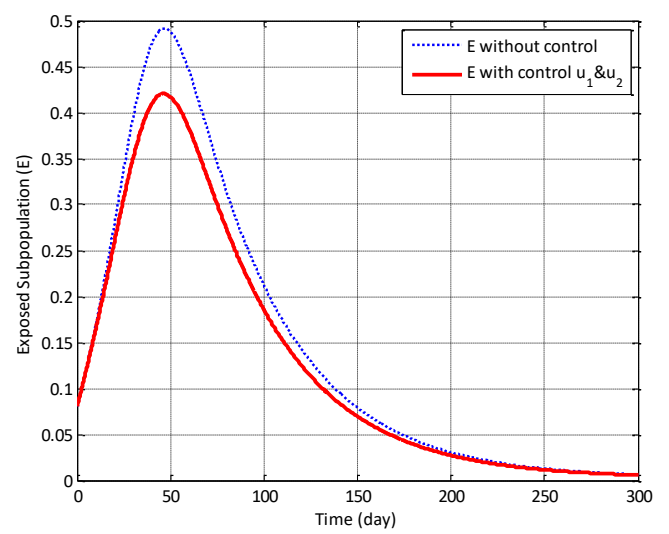

Figure 5. Density of Exposed Subpopulation (E) with Strategy Control $B$ and Without Control when $w_{1}=w_{2}=2000$ 
The small levels of vaccination control and treatment resulted in the number of infected subpopulations (I) not minimal, as the simulation results in Figure 6. That means, the population is still in an endemic condition where the spread of the disease occurs continuously.

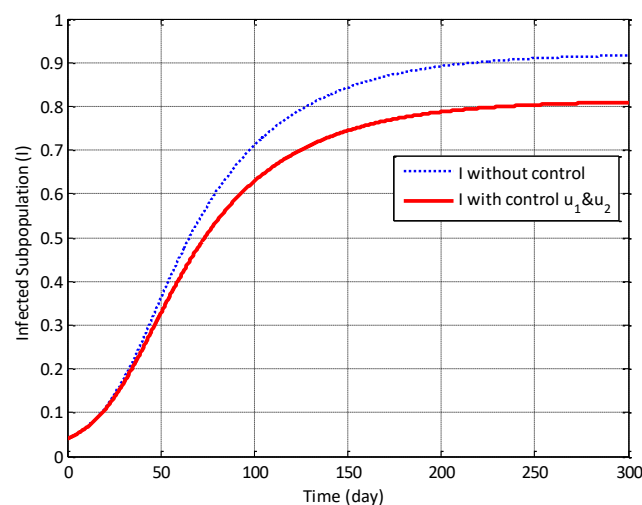

Figure 6. Density of Infected Subpopulation (I) with Strategy Control $B$ and Without Control when $w_{1}=w_{2}=2000$

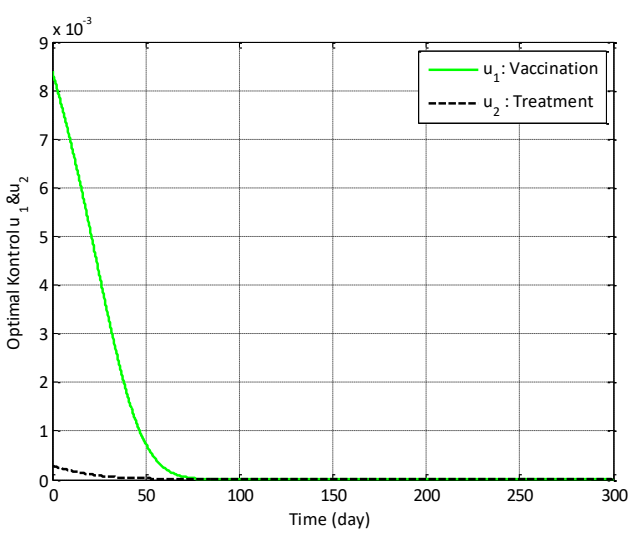

Figure 7. Vaccination control $u_{1}$ and treatment control $u_{2}$ are given every time (day) when $w_{1}=w_{2}=2000$

Furthermore, it is shown the effect of the weight of vaccination and treatment costs when it is reduced to ten times, i.e. equal to $w_{1}=w_{2}=2$. The simulation results in Figure 8 show that the number of exposed subpopulation is more minimum than the number of exposed subpopulation on other simulation results. This is similar to the change in the number of infected subpopulation in Figure 9. This is because the control level of vaccination and treatment is greater than the control level of other simulations, as shown in Figure 10. The vaccination control is given equal to 0.4322 and the treatment control is 0.3 at first. After the number of exposed and infected subpopulations decrease, the control level of vaccination and treatment is re- duced every time until it reaches zero value where the control is discontinued.

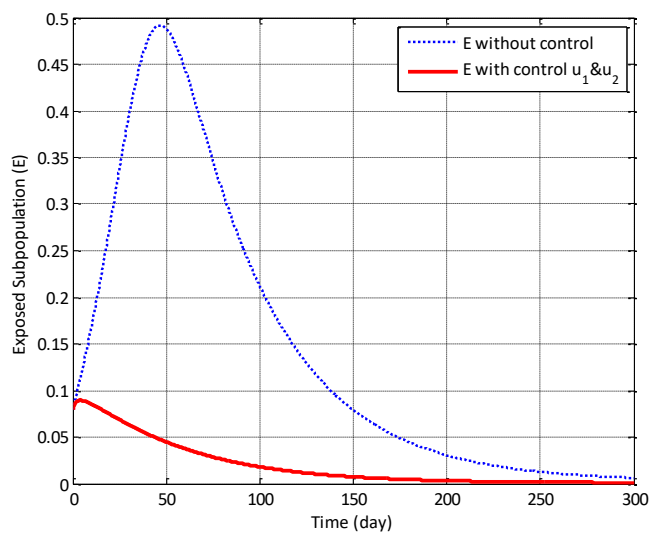

Figure 8. Density of Exposed Subpopulation (E) with Strategy Control B and Without Control when $w_{1}=w_{2}=2$

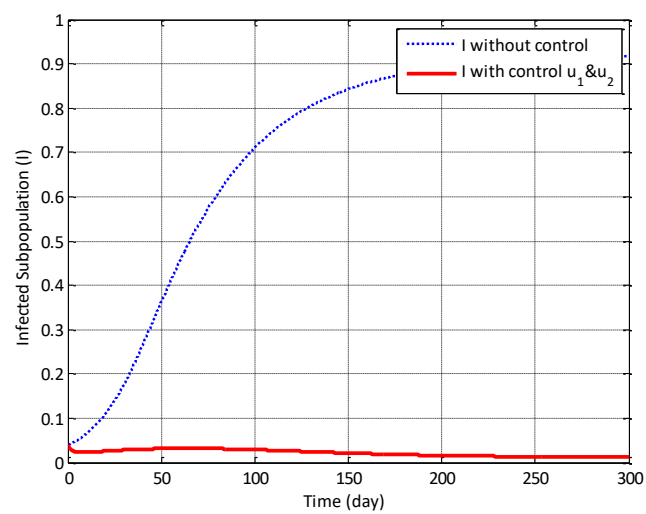

Figure 9. Density of Infected Subpopulation (I) with Strategy Control B and Without Control when $w_{1}=w_{2}=2$

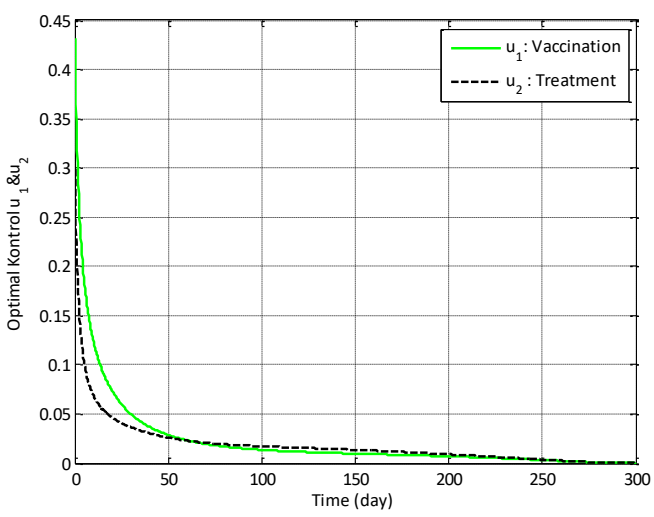

Figure 10. Vaccination control $u_{1}$ and treatment control $u_{2}$ are given every time (day) when $w_{1}=w_{2}=2$

The purpose of applying optimal control in this research is to minimize the objective functional $J$ in equation (3). The objective functional value is influenced by the increase and decrease of the number of exposed subpopulation, infected subpopulation, and controls. 
From Table 2, the minimum objective functional value is equal to 17.0064 . If the objective functional value is smaller then the control strategy applied in controlling the spread of SARS disease is more effective. Thus, it can be concluded that control combination strategy of vaccination and treatment with small cost weight is the most effective strategy.

Table 2. The objective functional value of each simulation for $t=300$

\begin{tabular}{cc}
\hline Strategy & Objective Functional \\
\hline $\begin{array}{c}\text { without control } \\
\left(u_{1}=u_{2}=0\right)\end{array}$ & 419.5444 \\
\hline $\begin{array}{c}\text { with control } u_{1} \\
\left(w_{1}=20\right)\end{array}$ & 141.8792 \\
\hline $\begin{array}{c}\text { with control } u_{2} \\
\left(w_{2}=20\right)\end{array}$ & 403.3616 \\
\hline $\begin{array}{c}\text { with control } u_{1} \text { and } u_{2} \\
\left(w_{1}=w_{2}=20\right)\end{array}$ & 88.3218 \\
\hline $\begin{array}{c}\text { with control } u_{1} \text { and } u_{2} \\
\left(w_{1}=w_{2}=2000\right)\end{array}$ & 376.7691 \\
\hline $\begin{array}{c}\left(w_{1}=w_{2}=2\right) \\
\text { with control } u_{1} \text { and } u_{2}\end{array}$ & 17.0064 \\
\hline
\end{tabular}

\section{CONCLUSION}

In this research, we conclude that the implementation of combination control strategy in the form of vaccination and treatment is more effective to reduce the number of exposed subpopulation (E) and infected subpopulation (I) than either the control strategy of vaccination only or the control strategy of treatment only. The weight of costs of vaccination and treatment controls can affect the levels of vaccination and treatment. If the weight of costs of vaccination and treatment are lower then the vaccination and treatment levels are greater. If the levels of vaccination control and treatment are getting bigger then the number of exposed and infected subpopulations are getting smaller. As a result, the objective functional value is getting smaller.

\section{ACKNOWLEDGEMENT}

We would like to express our gratitude to Biomathematics Group Study and LPPM, University of Brawijaya, Indonesia in accordance with the agreement of the research program implementation no: 730.8/UN10.C10/PN/2017 for financial support to this research.

\section{REFERENCES}

[1] Huang, G. 2016. Artificial infectious disease optimization: A SEIQR epidemic dynamic model-based function optimization algorithm. Swarm Evol. Comput. 27. 31-67.

[2] Kumar, A., P.K. Srivastava. 2017. Vaccination and treatment as control interventions in an infectious disease model with their cost optimization. Commun. Nonlinear Sci. Nu-mer. Simul. 44. 334-343.

[3] Gumel, A. B., S. Ruan, T. Day, J. Watmough, F. Brauer, P.V.D. Driessche, D. Gabrielson. C. Bowman, M.E. Alexander, S. Ardal, J. Wu, B.M. Sahai. 2004. Modelling strategies for controlling SARS outbreaks. Proc. Biol. Sci. 271(1554). 2223-2232.

[4] Surjawidjaja, J.E. 2003. Sindrom Pernafasan Akut Parah (Severe Acute Respiratory Syndrome/SARS): suatu epidemi baru yang sangat virulen. Kedokteran Trisakti. 2(22). 76-82.

[5] Yan, X., Y. Zou. 2008. Optimal and suboptimal quarantine and isolation control in SARS epidemics. Math. Comput. Model. 47(1-2). 235-245.

[6] World Health Organization. 2005. Combating emerging infectious diseases in the South-East Asia Region. February Edition.

[7] Otieno, G., J.K. Koske, J.M. Mutiso. 2016. Transmission dynamics and optimal control of malaria in Kenya. Discret. Dyn. Nat. Soc. Article ID 8013574. 27 pages.

[8] Sahu, G.P., J. Dhar. 2012. Analysis of an SVEIS epidemic model with partial temporary immunity and saturation incidence rate. Appl. Math. Model. 36(3). 908923.

[9] Li, J., Y. Yang, Y. Zhou. 2011. Global stability of an epidemic model with latent stage and vaccination. Nonlinear Anal. Real World Appl. 12(4). 2163-2173.

[10] Gumel, A.B., C.C. McCluskey, J. Watmough. 2006. An SVEIR model for assessing the potential impact of an imperfect anti-SARS vaccine. Math. Biosci. Eng. 3(3). 485-512. 